\title{
Cell Competition and Cooperation in Tissue Development
}

\author{
Jun Wang ${ }^{1 *}$, Brittany Egnot ${ }^{2}$ and Janet Paluh ${ }^{3}$ \\ ${ }^{1}$ Multiplex Biotechnology Laboratory, Cancer Research Center, Department of Chemistry, State University of New York, University at Albany, Albany, NY, USA \\ ${ }^{2}$ Bioengineering program, State University of New York, University at Albany, Albany, NY, USA \\ ${ }^{3}$ Nanobiosceince, Colleges of Nanoscale Science and Engineering, State University of New York Polytechnic Institute, Albany, NY, USA
}

${ }^{*}$ Corresponding author: Jun Wang, Multiplex Biotechnology Laboratory, Cancer Research Center, Department of Chemistry, State University of New York, University at Albany, Albany, NY, USA, Tel: 518442-4412; E-mail: jwang34@albany.edu

Received date: July 15, 2016; Accepted date: July 15, 2016; Published date: July 21, 2016

Copyright: ๑ 2016 Wang J, et al. This is an open-access article distributed under the terms of the Creative Commons Attribution License, which permits unrestricted use, distribution, and reproduction in any medium, provided the original author and source are credited.

\section{Editorial}

"Everything in nature is balanced", as taught in Tai Chi. Death and birth, light and dark, war and peace, happiness and sadness, are always coupled and occurring every day in human society. This balance is in fact universally existent in the physical world, even on the atomic scale between electrons and protons. On the cellular scale, this balance has started to become realized as an intangible principle behind tissue development. How should cellular competition and cooperation be defined? What are the biomarkers to distinguish these two cell interactions? What are the dynamic contributions of cell interactions in embryonic development, tumorigenesis, neurodegeneration and response to injury? Herein we summarize the most recent progress in cell interaction studies, to provide our review and perspective towards shedding light on more quantitative and determinative understanding of cell competition and cooperation. We focus on tumorigenesis mainly because it encompasses enriched interaction patterns as well as genetic mutation and evolution, and thus is an ideal model system.

Competition and cooperation are two intuitive terms that largely generalize cell interaction consequences. Competition is supposed to eliminate 'weak' or no longer needed cells and cooperation is supposed to maintain tissue homeostasis. Each can be subdivided into more specific interaction patterns (Figure 1) as in the case of tumorigenesis [1-3]. Cell competition as a selection process eliminates somatic genetic heterogeneity and promotes tissue fitness [4], which gives a positive impact in normal tissue development but also exerts the strongest negative impact in cancer progression where the most aggressive cancer subclones eventually thrive. The inhibition pattern of competition is commonly seen between cancer cells and immune cells through PD-1 and CTLA-4 pathways.

Other antagonistic patterns like predation and parasitism, which benefit one population by consuming the biomass of the other one, are commonly seen in systems other than tumors. Through competition, damaged, mutant, or otherwise unfit cells are prevented from contributing to the tissue and are instructed to die, whereas aggressive robustly dividing cells take over the microenvironment in the case of cancer; or, healthy cells benefit and populate the animal. Cooperation is the major format of cell interactions during tissue development, and mature cells in a tissue are geared toward cooperation to benefit the whole system. Commensalism is a type of cooperative interaction by which one population can benefit another without being affected itself, which could result in the proliferation of free-rider or cheater subclones that only take advantage of resources without making contributions. Cells can also behave synergistically to endow their offspring novel characteristics, or in a mutualistic manner where two or more populations cooperate and produce common goods to benefit all of the interacting parties. During tumor growth, multiple patterns of interactions are expected to co-exist simultaneously, and these patterns are expected to change dynamically along with the emergence of new phenotypes or subclones. Recent single cell sequencing efforts of tumors illustrate their heterogeneic makeup [5].

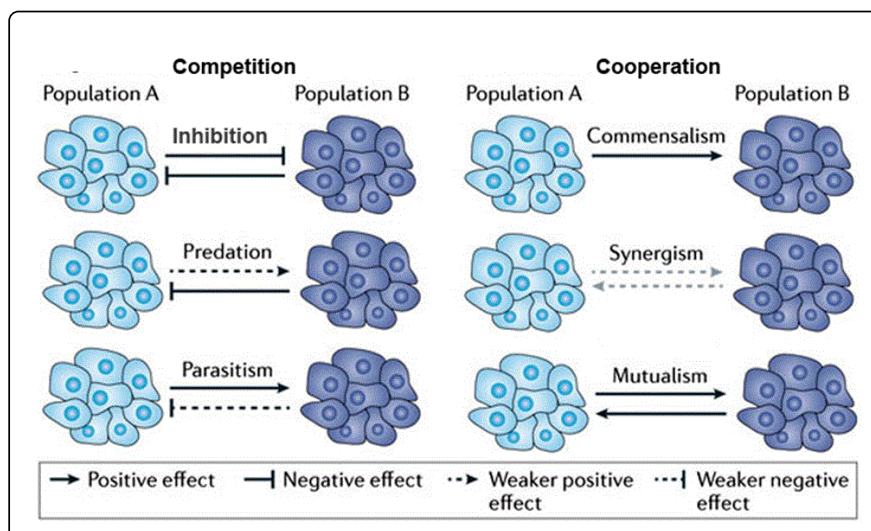

Figure 1: Types of ecological interactions among cell subpopulations [3].

Increasing attention is now being directed towards the cooperative behavior of tumor subclones that can influence disease progression [3], in the hope of disrupting cooperation and inducing tumor collapse. The tumor is a heterogeneous system with multiple coexisting subclones. Competition widely exists within close proximity for limited resources, resulting in complex interactions and selective sweeps in concordance with the 'survival of the fittest' aspect of Darwinian evolution. However, cooperation should always be coupled; otherwise the tumor microenvironment would lapse in growth, collapse and disappear. Interestingly, tumor progression is associated with the diminishing of fine architecture, which indicates the interaction dynamics are shifting closer to competition and the orders of homeostasis are continuously adjusted. Cooperative interactions, as a result of mutualistic or synergistic tendencies to benefit the tumor as a whole, were proposed to be one of the major drivers of persistent intratumor heterogeneity. The dynamic cooperation between subpopulations may be one of the treatment resistance mechanisms that compromise the effectiveness of targeted drug intervention. But, the cooperative behavior of cancer cells mostly remains as a theory with only a few experimental demonstrations and no mechanistic dissection of such cooperative interactions. It is reasonable to deduce that the 'hallmarks of cancer' may be acquired collectively, by multiple collaborative subclones, instead of one subclone accumulating all the necessary mutations [6]. For example, a high degree of heterogeneity can imply poor disease prognosis in cancer patients $[7,8]$. In fact, 
cooperation of subpopulations has been observed before to promote tumor growth, such as basal Wnt $1^{\text {low }}$ subclones and luminal Wnt $1^{\text {hi }}$ subclones [9], CD29hi CD24hi tumor initiating cells and more differentiated $\mathrm{CD} 29^{\text {hi }} \mathrm{CD} 24^{\text {low }}$ mesenchymal populations [10], IL-11 overexpressing cells and FIGF overexpressing cells [11], amongst others.

As above mentioned, competition and cooperation are extremely difficult to quantify. In the past years, we have developed a microfluidic technology and demonstrated the dynamics of cell interactions to signaling outputs. For cancer cell interactions of the same phenotype via these high-throughput microchips we found that certain cell types containing the EGFRvIII mutation exhibit self-inhibition when those cells are less than 80 microns apart, but self-activate at large separation distances (Figure 2). The EGFRvIII mutation is known to result in persistent cell growth and proliferation, but cell interactions impact the outcome. Therefore, those self-inhibitory cells may avoid targeted drugs inhibition through coupled cell interactions. Other cancer cell types exhibit very different behaviors as a function of cell-cell separation. Our recent work on glioblastoma demonstrates a similar phenomenon. In these associated studies we show that IL-6 and VEGF, which function as promoting cancer cell growth and division, were highly expressed in the in vitro culture medium and the in vivo tumor specimen [12]. Our study infers that cell interactions may play a nontrivial, if not the major, role in determining cellular functions and phenotypes as well as tumor hierarchy. This result also implies that cancer cell interactions within a tumor may shield them as drug targets [9].

Protein signaling is often linked with cell interaction behaviors and is a reliable indicator of interaction patterns. Signaling is the first responder to any cellular behaviors, including cell interactions. For example, Wnt signaling has been shown as an important participant in cooperative interactions of tumor subclones [9]. Basal Wnt ${ }^{\text {low }}$ subclones and luminal Wnthi subclones exhibit clearly cooperative interactions, and both are required for tumorigenesis but there is higher dependence on Wnt production by the luminal subclones. This is the first study demonstrating that intratumoral heterogeneity and tumor cell hierarchy can be caused and maintained by cell interactions, besides clonal evolution and stochastic transition in conventional knowledge. Although the cooperative behavior of tumor subclones has been demonstrated as the key to tumorigenesis, when and how distinct subclones emerge in the course of tumor progression is still unknown [9]. In the glioblastoma study, we discovered that there is a populationwide equilibrium between EGFRvIII ${ }^{+}$cells and EGFRvIII ${ }^{-}$cells, and the continued culture after removal of either of them will restore the equilibrium [12]. The presence of this mutant signaling protein results in constant activation of the PI3K pathway and accelerates tumor growth. EGFRvIII cells in a tumor cooperate with their neighbors through exchange of cytokines IL-6 and LIF. This phenomenon also infers that through cooperation, a minor subpopulation can drive the accelerated growth of the entire tumor and thereby actively maintain cell heterogeneity within a tumor. Investigation of the signaling networks of interacting EGFRvIII cells implies that these aggressive cancer cells swiftly shift between cooperative and competitive modes, depending on cell-cell distance as measured in a confined small chamber.

How do we quantitatively understand cell competition and cooperation? In human society, these interactions can be best understood by game theory. Since a single cell is the basic unit of life, game theory has also been thought to be applicable to individual cells in a tissue. Although there is not yet sufficient experimental data to support this model, mainly due to cellular heterogeneity, evolutionary game theory is the most accepted mechanism for explaining population dynamics of cancer cell subclones [13-18]. The derived equilibria of populations are based on Nash equilibria where the competing players receive benefits by cooperating to a certain degree, and cancer cells make rational choices when interacting with others. If a population of irrational cells engages in some (genetically determined) strategy (they did not choose), the final state will coincide with the strict Nash equilibrium that a pair of rational payoff maximizers will choose when they interact once according to a game model with the same payoff matrix $[19,20]$. That means, different from the prisoner's dilemma, individual cells may not necessarily make the best choices for themselves because of the evolutionary process, but instead eventually they will achieve equilibrium through competition and cooperation.

\section{Future of Cell Interaction Studies}

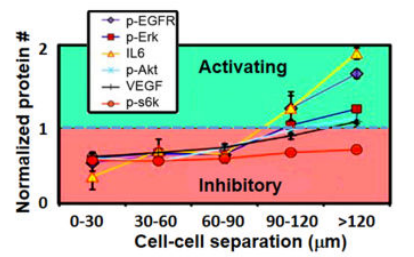

Figure 2: Transition between inhibitory state and activating state by varying cell-cell distances, evaluated by oncogenic protein levels $[1,2]$.

Much work still needs to be done before knowledge of cell interactions can be harnessed to have a high impact on our understanding and treatment of diseases affecting human health and in designing in vitro microenvironment niches [21]. The major issue that impedes this field is the lack of quantitative data at the subpopulation level that are directly associated with competition and cooperation patterns. When it comes to subpopulation genome wide genomic characterization, single-cell techniques are always the first choice; however, all single-cell tools have the same weakness, which is the loss of system integrity as a tissue is not simply additive with the number of individual cells. New tools for generating data of cell subset interactions are emerging, which are expected to revolutionize our understanding of normal developmental biology, disease, injury and cancer biology fields. The theoretical framework beyond evolutionary game theory will follow up quickly, which has been exemplified in our work on predicting tumor architecture. With high-throughput quantitative data, principles borrowed from physics and mathematics could be applied to precisely predict biological behaviors. We have already taken the initiative to employ thermodynamics to predict brain tumor architecture based on high-throughput pairwise interaction data [2]. In the near future, we expect to understand more about the fundamentals of biological systems and perhaps even more exciting, to begin to manipulate various cell interactions to facilitate disease therapies. 
Citation: Wang J, Egnot B, Paluh J (2016) Cell Competition and Cooperation in Tissue Development. J Tissue Sci Eng 7: 131. doi: $10.4172 / 2157-7552.1000131$

Page 3 of 3

\section{References}

1. Wang J, Tham D, Wei W, Shin YS, Ma C, et al. (2012) Quantitating cellcell interaction functions with applications to glioblastoma multiforme cancer cells. Nano Lett 12: 6101-6106.

2. Kravchenko-Balasha N, Wang J, Remacle F, Levine RD, Heath JR (2014) Glioblastoma cellular architectures are predicted through the characterization of two-cell interactions. Proc Natl Acad Sci U S A 111: 6521-6526.

3. Tabassum DP, Polyak K (2015) Tumorigenesis: It takes a village. Nature reviews Cancer 15: 473-483.

4. Johnston LA (2014) Socializing with MYC: Cell competition in development and as a model for premalignant cancer. Csh Perspect Med 4 .

5. Navin NE (2015) The first five years of single-cell cancer genomics and beyond. Genome Research 25: 1499-1507.

6. Axelrod R, Axelrod DE, Pienta KJ (2006) Evolution of cooperation among tumor cells. P Natl Acad Sci USA 103: 13474-13479.

7. Notta F, Mullighan CG, Wang JCY, Poeppl A, Doulatov S, et al. (2011) Evolution of human BCR-ABL1 lymphoblastic leukaemia-initiating cells. Nature 469: 362-367.

8. Almendro V, Cheng YK, Randles A, Itzkovitz S, Marusyk A, et al. (2014) Inference of tumor evolution during chemotherapy by computational modeling and in situ analysis of genetic and phenotypic cellular diversity. Cell Rep 6: 514-527.

9. Cleary AS, Leonard TL, Gestl SA, Gunther EJ (2014) Tumour cell heterogeneity maintained by cooperating subclones in Wnt-driven mammary cancers. Nature 508: 113-117.

10. Mateo F, Meca-Cortes O, Celia-Terrassa T, Fernandez Y, Abasolo I, et al. (2014) SPARC mediates metastatic cooperation between CSC and nonCSC prostate cancer cell subpopulations. Mol Cancer 13: 237-239.
11. Marusyk A, Tabassum DP, Altrock PM, Almendro V, Michor F, et al. (2014) Non-cell-autonomous driving of tumour growth supports subclonal heterogeneity. Nature 514: 54-58.

12. Nathanson DA, Gini B, Mottahedeh J, Visnyei K, Koga T, et al. (2014) Targeted therapy resistance mediated by dynamic regulation of extrachromosomal mutant EGFR DNA. Science 343: 72-76.

13. Archetti M, Ferraro DA, Christofori G (2015) Reply to Gerlee and Altrock: Diffusion and population size in game theory models of cancer. P Natl Acad Sci USA 112: E2744-E2744.

14. Pacheco JM, Santos FC, Dingli D (2014) The ecology of cancer from an evolutionary game theory perspective. Interface Focus 4.

15. Archetti M (2013) Evolutionary game theory of growth factor production: Implications for tumour heterogeneity and resistance to therapies. Brit J Cancer 109: 1056-1062.

16. Basanta D, Scott JG, Fishman MN, Ayala G, Hayward SW, et al. (2012) Investigating prostate cancer tumour-stroma interactions: Clinical and biological insights from an evolutionary game. Brit J Cancer 106: 174-181.

17. Kianercy A, Veltri R, Pienta KJ (2014) Critical transitions in a game theoretic model of tumour metabolism. Interface Focus 4.

18. Basanta D, Simon M, Hatzikirou H, Deutsch A (2008) Evolutionary game theory elucidates the role of glycolysis in glioma progression and invasion. Cell Proliferat 41: 980-987.

19. Dingli D, Chalub FACC, Santos FC, Van Segbroeck S, Pacheco JM (2009) Evolutionary game theory: lessons and limitations, a cancer perspective Reply. Brit J Cancer 101: 2062-2063.

20. Huang WN, Haubold B, Hauert C, Traulsen A (2012) Emergence of stable polymorphisms driven by evolutionary games between mutants. Nat Commun 3.

21. Paluh JL, Dai G, Chrisey DB (2011) In search of the Holy Grail: Engineering the stem cell niche. European Pharmaceutical Review 16. 